\title{
Personería gremial y conflicto colectivo en Argentina: la experiencia de los Metrodelegados (2015-2019)
}

\author{
Recibido: 20 de abril de 2020 • Aprobado: 17 de junio de 2020 \\ https://doi.org/10.22395/ojum.v20n4la10
}

\author{
Jorge Rubén Afarian \\ Universidad de Buenos Aires, Buenos Aires, Argentina \\ jafarian@derecho.uba.ar \\ https://orcid.org/0000-0002-9350-2768
}

\section{RESUMEN}

En el presente artículo analizaremos las correlaciones, imbricaciones y desconexiones entre el ámbito jurídico y el conflicto en los reclamos sobre condiciones de trabajo, concretamente a través de la experiencia de la solicitud de personería gremial por parte de los Metrodelegados del subterráneo de la Ciudad Autónoma de Buenos Aires. A partir de la historia particular del gremio, se indagará el grado de influencia del conflicto colectivo en la adquisición de derechos laborales y se señalará el rol que adoptan las normas en aquel proceso.

Realizaremos un breve recorrido por la historia del sindicato y la normativa aplicable a las relaciones colectivas a partir de un análisis cualitativo que incluye notas periodísticas, normas, declaraciones de referentes del gremio y registros etnográficos de actos públicos convocados por el sindicato. Por último, tomaremos el caso específico del asbesto hallado en las formaciones del subte a principios de 2018, mineral de alta toxicidad y perjudicial para la salud de los trabajadores y usuarios. Recurriremos a este caso a modo de experiencia testigo de la problemática entre el campo jurídico y el conflicto colectivo de trabajo. Una de las principales conclusiones a las que arribamos es que, en este caso, el conflicto constituye una herramienta esencial para la obtención de derechos laborales, más allá de contar o no con la personería gremial. Sin perjuicio de ello, se apela y utiliza la normativa para afianzar el poder "real", en los hechos, que posee el sindicato de subterráneo.

Palabras clave: conflicto colectivo; personería gremial; Metrodelegados. 


\section{Union Personality and Collective Conflict in Argentina: the Experience of the Metrodelegates (2015-2019)}

\section{ABSTRACT}

In this article, we will analyze the correlations, overlaps and disconnections between the legal field and the conflict in claims about working conditions, specifically through the experience of the application for union status by the Metrodelegates of the subway of the autonomous city of Buenos Aires. From the particular history of the union, we will investigate the degree of influence of the collective conflict in the acquisition of labor rights, pointing out the role that the rules adopt in that process.

We will make a brief journey through the history of the union and the regulations applicable to collective relations, based on a qualitative analysis that includes journalistic notes, regulations, statements from leaders of the union and ethnographic records of public events called by the union. Finally, we will take the specific case of asbestos found in the subway formations in early 2018, a highly toxic mineral that is harmful to the health of workers and users. We will use this case as a witness experience of the problems between the legal field and the collective labor conflict. One of the main conclusions we reached is that, in this case, the conflict constitutes an essential tool for obtaining labor rights, regardless of whether or not it has union status. Without prejudice to this, the regulations are appealed and used to consolidate that "real" power, in fact, that it possesses in the underground union.

Keywords: collective conflict; union personality; Metrodelegates.

\section{Estatuto sindical e conflito colectivo na Argentina: a experiência dos Metrodelegados (2015-2019)}

\section{RESUMO}

Neste artigo vamos analisar as correlações, sobreposições e desconexões entre a esfera jurídica e o conflito nas reivindicações sobre as condições de trabalho, especificamente através da experiência da candidatura à filiação sindical pelos Metrodelegados do Metro da Cidade Autónoma de Buenos Aires. A partir da história particular do sindicato, será investigado o grau de influência do conflito colectivo na aquisição de direitos laborais e será apontado o papel adoptado pelas regras nesse processo.

Faremos um breve passeio pela história do sindicato e pelos regulamentos aplicáveis às relações colectivas a partir de uma análise qualitativa que inclui notas jornalísticas, regras, declarações de dirigentes sindicais e registos etnográficos de eventos públicos convocados pelo sindicato. Finalmente, tomaremos o caso específico do amianto encontrado nos comboios do metro no início de 2018, um mineral altamente tóxico que é prejudicial para a saúde dos trabalhadores e utilizadores. Recorreremos a este caso como testemunha da experiência da problemática entre o campo jurídico e o conflito colectivo de trabalho. Uma das principais conclusões a que chegámos é que, neste caso, o conflito é um instrumento essencial para a obtenção de direitos laborais, independentemente de terem ou não filiação sindical. Sem prejuízo disso, os regulamentos são apelados e utilizados para reforçar o poder "real", de facto, que o sindicato do metro tem.

Palavras-chave: conflito colectivo; estatuto sindical; Metrodelegados. 


\section{INTRODUCCIÓN}

Este trabajo se inserta en una beca de investigación doctoral financiada por la Universidad de Buenos Aires y en el proyecto de investigación UBACyT: Las peticiones de los sectores populares a la convención constituyente de 1949: prácticas y expectativas ante la reforma constitucional con número 20020190200038BA, a cargo de la profesora Leticia Vita. En este contexto, he presentado avances de mi trabajo en diversos encuentros académicos y jornadas, a la vez que varios resultados y reseñas de este tema particular han sido publicados en diversas revistas y espacios de difusión (Afarian, 2018, 2019a, 2019b).

Dicho esto, comenzaré mi exposición. La personería gremial (PG) es un derecho exclusivo que se otorga administrativamente mediante resolución del Ministerio de Trabajo, Empleo y Seguridad Social a la asociación sindical de trabajadores más representativa, de acuerdo con su actividad, trabajadores afiliados cotizantes y el ámbito territorial de representación de la asociación que lo solicita. Su trámite se encuentra regulado específicamente en la Ley 23.551 (1988) de asociaciones sindicales, junto con sus decretos reglamentarios. A partir de aquel reconocimiento, la representatividad se entrecruza con la representación, y esta última a partir del instituto de la personería gremial.

Al ser una particularidad especial a la que pocos sindicatos tienen acceso, y muchos menos pueden disputar y ganar ese derecho a una asociación sindical que ya la posea, la asociación sindical que lo adquiere cuenta con un extenso listado de derechos y privilegios de carácter colectivo. Entre ellos incluimos la representación de los intereses colectivos ante los empleadores, el Estado y los organismos internacionales, la negociación y firma de convenios colectivos de trabajo, la creación de obras sociales sindicales, entre muchos otros.

Del lado contrario, y a pesar de que el artículo 14 bis de la Constitución nacional (2010) establece un sistema abierto de representación sindical, "con la simple inscripción en un registro especial" y un derecho de asociación gremial amplio expresado por el Convenio 87 (OIT, 1948) y el Convenio 98 (OIT, 1949, arts. 1 y 2) sobre libertad sindical, la ley argentina opta por otorgar derechos exclusivos solo a los sindicatos más representativos y, preferentemente, por rama de actividad.

Relacionado con ello, la Asociación Gremial de Trabajadores del Subte y Premetro (AGTSyP, también conocida como los Metrodelegados) desplazó de la PG a la Unión Tranviarios Automotor (UTA) a finales de 2015, expediente que había iniciado en julio de 2013. Sin embargo, en marzo de 2017 la Sentencia 110121 (2017) de la Cámara Nacional de Apelaciones del Trabajo (Sala II) deja sin efecto la adjudicación de la PG, la devuelve a la UTA. Sustenta su decisión en argumentos formales y procesales que, desde nuestra visión, son cuestionables. Este fallo fue confirmado por la Sentencia CNT 77335/2014/CSl (2018) de Corte Suprema de Justicia de la Nación a mediados de marzo. 
Casi al mismo tiempo, y luego de la decisión de la Corte Suprema, se multiplican los conflictos colectivos en aquella actividad en reclamo de acuerdos paritarios y mejores condiciones de salud y seguridad, pero también a raíz de numerosas desvinculaciones y despidos. A partir de ello, nos preguntamos en qué medida el conflicto legitima la acción sindical del gremio, más allá de la personería gremial como la pretendida herramienta jurídica y, en teoría, más completa en el mundo de las relaciones colectivas del trabajo. Este instituto impera en nuestra cultura jurídica desde hace más de setenta años.

\section{BREVE HISTORIA DE LOS METRODELEGADOS}

"Che Mauricio, che Mauricio', no te lo decimos más, con papeles o sin papeles, te paramos la ciudad". Cántico durante la conferencia de prensa en el subterráneo, a raíz de la perdida de la personería gremial. (AGTSyP, 2018)

La historia de la AGTSyP, mejor conocida desde su origen como los Metrodelegados posee diversas aristas y características propias que merecen ser destacadas. Desde su constitución como grupo independiente — disconforme con la dirigencia sindical de la UTA (Unión Tranviarios Automotor) a la que pertenecían - hasta la actualidad, esta organización posee una trayectoria de las más particulares en la historia gremial de la Argentina.

El surgimiento del cuerpo de delegados del subterráneo (como se los conocía cuando aún formaban parte de la UTA) no es casual ni accidental, sino que responde a una lógica económica y social que afectó las bases mismas de la estructura laboral, y le imprimió caracteres regresivos a las relaciones laborales de producción. En Argentina, el más claro ejemplo lo constituye la política de privatizaciones de empresas y servicios públicos durante los dos períodos presidenciales de Carlos Menem en el período 1989-1999.

Sin embargo, aquellas no fueron exclusivas de dicho período, puesto que este proceso se remonta hasta la dictadura cívico-militar de 1976-1983 (Cortés y Marshall, 1991; Verbitsky y Bohoslavsky, 2013), e incluso desde la presidencia de Arturo Frondizi a finales de la década de los cincuenta con la privatización del frigorífico Lisandro de la Torre que dio lugar a la toma y posterior represión en el lugar de trabajo por parte de las fuerzas de seguridad (Pontoriero, 2015; Salas, 1990).

La Ley 23.696 (1989) de emergencia económica, sancionada a finales de 1989, comenzó un proceso sistemático de retirada del Estado de los servicios públicos mediante la concesión a manos privadas de numerosas empresas. El servicio de transporte subterráneo no permaneció incólume frente a aquellos embates, sino que,

Se refiere al presidente de la Argentina de aquel momento, Mauricio Macri, quien previamente había sido jefe de Gobierno de la ciudad de Buenos Aires (2007-2015). 
por sus características particulares, fue uno de los principales afectados por dichas "reformas" estatales.

En efecto, a principios de enero de 1994, el subterráneo de la Ciudad de Buenos Aires fue concedido a la empresa privada Metrovías S. A. Ello trajo consigo una reformulación de las prioridades de la nueva dirección, lo que generó también modificaciones en la estructura productiva de dicha entidad. En lugar de aplicarse el esquema de derechos y garantías del trabajo estatal, comenzó a aplicarse el régimen de trabajo privado. De esta manera, se hizo hincapié en contratos con limitadas garantías laborales y de estabilidad en el puesto de trabajo.

Concretamente, Metrovías inició una serie de medidas que modificaron tanto la estructura y modalidades de la actividad como el número de trabajadores bajo relación de dependencia. Así, por ejemplo, se produjeron una serie de despidos que se encubrieron mediante "retiros voluntarios" que dieron lugar, en muchos casos, a recontrataciones a través de empresas de servicios eventuales. Estas constituyen características particulares en casi todas las ramas productivas que fueron privatizadas. En escasos meses, se despidieron alrededor de mil quinientos trabajadores y trabajadoras del subterráneo (Basualdo et al., 2015; Ventrici, Compáñez y Vocos, 2012).

En términos de condiciones de trabajo, el servicio fue despojado de su característica de insalubre a través de convenciones colectivas de trabajo, lo que a su vez trajo aparejado la elevación mediante convenio colectivo de trabajo de la carga horaria de la actividad, de las seis horas diarias a las ocho horas diarias. Por otro lado, la empresa decidió tercerizar y subcontratar servicios esenciales, tales como la seguridad, limpieza, reparación, entre muchos otros (Arias, 2015; Arias y Haidar, 2008; Arias, Diana Menéndez y Salgado, 2013; Bouvet, 2008; Castillo et al., 2007; Cresto, 2010, 2012; Montes Cató y Ventrici, 2010; Ventrici, Compáñez y Vocos, 2012; Vocos, 2008).

Ante este panorama, el sindicato que debía velar por los derechos laborales y sociales (la UTA) no asumió sus compromisos con dicho colectivo, sino que ofreció concesiones vejatorias de importantes derechos laborales, permitió la entrada de numerosas contrataciones flexibles (los convenios colectivos de trabajo de empresa 121/94 y 384/99 modificatorios del 41/75 son claros ejemplos) y censuró cualquier intento de resistencia en el lugar de trabajo, tanto en materia de conflicto como en la creación de nuevas agrupaciones (Bouvet, 2008; Cresto, 2010, 2012; Ventrici, Compáñez y Vocos, 2012).

La historia de los Metrodelegados comienza en esta etapa de cambios estructurales de las relaciones del trabajo como un subgrupo "de base" con orientación combativa de izquierda, proveniente de partidos socialistas como el Movimiento al Socialismo (MAS) y el Partido de los Trabajadores Socialistas (PTS), cuyas estructuras orgánicas eran muy diferentes a las de la UTA. 
Como advirtiéramos más arriba, los comienzos de los Metrodelegados estuvieron signados por la clandestinidad, puesto que cualquier opinión en contra de la burocracia sindical representada en la UTA o cualquier intento de agremiación independiente atentaban con la precaria situación laboral de los empleados de la empresa. El objetivo era resguardarse del despido y las represalias en un momento en el que cualquier organización opositora no contaba con la legitimidad y fuerza necesarias para otorgar suficientes garantías de acción y protección a los representantes (Bouvet, 2008; Dantón Bermúdez, 2013; Trajtemberg y Battistini, 2015; Ventrici, 2011; Ventrici, Compáñez y Vocos, 2012).

Esta coyuntura, compuesta de reuniones fuera del horario de trabajo y en lugares ajenos a él, se modificó radicalmente en febrero de 1997 a raíz del despido injustificado de un joven conductor de la línea E. La desvinculación se debió a un desperfecto mecánico producto de la asignación de una tarea para la que no estaba debidamente capacitado. Ello produjo la primera huelga (conocida como el Paro de Varela) a Metrovías sin el consentimiento de la dirigencia de la UTA. Luego de la conciliación obligatoria entre la UTA y el Ministerio de Trabajo, el trabajador fue reincorporado.

Irónicamente, una de las mayores razones de la conformación de cuerpo de delegados era el nuevo grupo de trabajadores jóvenes, muchos de ellos contratados a través de la modalidad de "bolsa de trabajo". Esta estrategia era utilizada por el empresa para hallar mano de obra carente de experiencia y adaptable a las condiciones contractuales laborales promovidas por el Estado, especialmente a partir de la Ley 24.013 (1991). Precisamente los jóvenes devinieron en actores de importancia trascendental en la lucha por la mejora de las condiciones de trabajo.

La medida adoptada en 1997 fue una de las primeras manifestaciones de la estrategia de confrontación, propia de los grupos de izquierda, que con el tiempo se convirtió en uno de los signos distintivos de los Metrodelegados que se mantuvo hasta nuestros días (Arias y Haidar, 2008; Ventrici, 2011). A su vez, logró dar visibilidad a la organización. A partir de aquel momento se alejó de su estatus de clandestinidad y pasó a adoptar medidas de conflicto y reclamo directos.

Tanto es así que en los años posteriores, especialmente luego de la crisis socioeconómica del 2001, los Metrodelegados actuaron como uno de los principales interlocutores entre la dirigencia empresarial y los reclamos de los trabajadores. Por ejemplo, en 2004 mediante un nuevo acuerdo colectivo de trabajo, el subterráneo regresó a la jornada de seis horas, que hasta ese momento era de ocho horas (pactado en el convenio colectivo de trabajo luego de su privatización en 1994).

Finalmente, a principios de septiembre de 2008, el cuerpo de delegados del subte, los Metrodelegados pasaron a ser un sindicato formalmente constituido y redactaron su estatuto fundacional y denominándose AGTSyP. Luego de numerosos pedidos, 
marchas y paros de actividades reclamando la inscripción gremial en el Ministerio de Trabajo (AGTSyP, 2009a, 2009b), finalmente en noviembre de 2010 se les otorga la personería jurídica. Es aquí donde comienza la solicitud de personería gremial, iniciada a finales de julio de 2013.

\section{LA PERSONERÍA GREMIAL (2013-2018)}

Como expresáramos al principio de nuestro trabajo, el instituto de la personería gremial es un conjunto de derechos otorgados por el Ministerio de Trabajo, Empleo y Seguridad Social de la nación a partir de una audiencia de cotejo de trabajadores y trabajadoras afiliadas y cotizantes, en la que cada sindicato debe demostrar una mayor representatividad. Aquella es guiada por un criterio funcional (actividad concreta desplegada por el colectivo), profesional (actividad, profesión o empresa) y territorial (sea nacional, provincial o regional). En este sentido, los sindicatos con esta característica cuentan con derechos exclusivos que no poseen otros sindicatos simplemente inscriptos y que incluso pueden pertenecer al mismo ámbito de actuación (Corte, 1994; Recalde, 2015).

En este sentido, siguiendo a Drolas (2003) y a Trajtemberg et al. (2012) y Ventrici (2016), la representación (es decir, la personería gremial que se otorga) proviene del Estado a través de este reconocimiento y otorgamiento de derechos específicos al sindicato. Sumado a ello, aunque aquella representación muchas veces coincida con el reconocimiento de tal sindicato en los lugares de trabajo (la representatividad), esto no siempre sucede. Entonces estamos en presencia de un poder concreto, esto es, "real" (expresado en este caso en el conflicto) y un poder normativo "formal" (expresado en la personería gremial).

La personería gremial fue instituida a partir del Decreto-Ley 23.852 (1945) durante el Gobierno de facto de Edelmiro Farrell, y luego ratificada con la Ley 14.250 (1953) sobre convenciones colectivas de trabajo en la última etapa de la presidencia de Perón. Su procedimiento de obtención y consecuencias casi no variaron en más de siete décadas (Afarian y Pasarín, 2019). Por el contrario, todas las leyes que actualmente rigen se concentran en justificar y motorizar el sistema y aseguran su legitimidad tanto política como jurídica. Lo mismo sucede con las sentencias judiciales que tampoco cuestionan la estructura, sino que realizan "aperturas" parciales (Sentencia 331:2499, 2008; Sentencia 332:2715, 2009).

Luego de dictaduras militares cada vez más sangrientas, durante los años ochenta se produce el primer intento institucionalizado de "democratización sindical" durante el gobierno de Raúl Alfonsin que aspiraba a un mayor recambio de las autoridades y delegados. Este intento, sin embargo, no tuvo éxito. Finalmente, se sanciona la Ley 23.551 (1988) de asociaciones sindicales, en el que se reconfirma el sistema de mayores derechos provenientes de la personería gremial, principalmente en el artículo 31 de dicho cuerpo normativo. Aquí la ley diferencia entre sindicatos "simplemente 
inscriptos" y sindicatos "con personería gremial". Estos últimos, además de contar con todos los derechos de un sindicato simplemente inscripto, poseen otras atribuciones específicas, que son las siguientes:

1 Defender y representar ante el Estado y los empleadores los intereses individuales y colectivos de los trabajadores.

2 Intervenir en negociaciones colectivas, firmar convenciones colectivas de trabajo y vigilar el cumplimiento de la normativa laboral y de seguridad social.

3 Administrar sus propias obras sociales y, según el caso, participar en la administración de las creadas por ley o por convenciones colectivas de trabajo.

Criticada desde la sanción misma de la nueva ley por diversos organismos internacionales como la OIT (2006) a través de la Comisión de Expertos en la Aplicación de Convenios y Recomendaciones, la personería gremial continúa siendo uno de los hitos principales y un rasgo distintivo del llamado "modelo sindical argentino" (Corte, 1994; Tribuzio, 2012; Recalde, 2015) consistente en una pluralidad sindical con unicidad promocionada.

En lo que respecta a los Metrodelegados, el 31 de julio de 2013 estos solicitaron el desplazamiento de la personería gremial de acuerdo con las previsiones de los artículos 25 y 28 de la Ley 23.551 (1988) y del artículo 21 del Decreto 467 (1988). Allí se establece que la asociación sindical que pretenda tales derechos debe acreditar, además del $20 \%$ que se exige para la adquisición originaria, un $10 \%$ adicional para el desplazamiento, con lo que se precisaría una base de afiliados mínima del $30 \%$. Por ello, son contadas las veces en que se produjo el desplazamiento de la personería gremial hacia otro sindicato.

Ahora bien, en la audiencia de cotejo de afiliados cotizantes de la AGTSyP, celebrada el 28 de noviembre de 2013, se probó la afiliación de casi el $60 \%$ de los trabajadores que prestan servicios en relación de dependencia en el transporte público de subterráneo. La UTA no compareció a la audiencia. Después de numerosos pedidos de archivo y desestimación de peticiones por parte de la UTA, se designó una nueva audiencia de cotejo de representatividad entre los registros de la AGTSyP y la UTA en julio de 2014.

Como consecuencia de un recurso jerárquico interpuesto por la UTA, que fue rechazado por el Ministerio de Trabajo, se designó otra audiencia para el 22 de diciembre de 2014. Después de diversas presentaciones del mencionado sindicato a los efectos de suspender la sustanciación de la audiencia, junto con la interposición del recurso extraordinario ante la Corte Suprema de Justicia de la Nación que fueron rechazados, se designó una nueva audiencia para el 12 de noviembre de 2015.

En aquella oportunidad, solo compareció la AGTSyP, que acreditó la afiliación en los mismos valores que los de la audiencia del 28 de noviembre de 2013. Por su parte, 
la Dirección Nacional de Asociaciones Sindicales fijó una nueva y última audiencia para el día 1 de diciembre de 2015. La AGTSyP solicitó la revocación de la nueva audiencia de cotejo de representatividad que fue aceptada y se le otorgó la personería gremial mediante la Resolución 1601 (2015), publicada en el Boletín Oficial el día 1 de diciembre de 2015. Cabe agregar que la personería le fue adjudicada días antes de la finalización del Gobierno de Cristina Fernández de Kirchner, cuyo signo político había sido derrotado en elecciones por su principal opositor, Mauricio Macri.

Poco más de un año después, el día 6 de marzo de 2017, la Sala II de la Cámara Nacional de Apelaciones del Trabajo, con base en un recurso de apelación directo basado en el artículo 62 de la Ley 23.551 de Asociaciones Sindicales (1988) interpuesto por la Sentencia 110121 (2017), revocó la Resolución 1601 (2015) de otorgamiento de la personería gremial. Basó su decisión en el fundamento de que el proceso seguido en sede administrativa no respetó el derecho de defensa de la UTA con la intensidad que exige la ley sindical, y que las pruebas valoradas en la resolución de desplazamiento de la personería gremial habían sido obtenidas sin la participación de aquella asociación sindical (todo ello a pesar de las repetidas incomparecencias del sindicato a las audiencias fijadas).

Por otro lado, el tribunal consideró que la autoridad administrativa no articuló ningún mecanismo procedimental a fin de otorgar la posibilidad de participar en la producción de tales pruebas, lo que afectó la bilateralidad del proceso. La resolución cuestionada tiene acreditados los recaudos para el desplazamiento de la personería gremial sin la intervención de la UTA, tanto en el caso de determinar el universo de trabajadores representados como en la producción de las pruebas pertinentes a fin de garantizar la defensa en juicio.

De acuerdo con Gianibelli (2017), "la Cámara expresó que no existe una norma que contemple el apercibimiento por el cual la incomparecencia a la audiencia de cotejo de representatividad lleve a presumir la intensidad de la representación de la peticionaria". Esto quiere decir que, sumado al arrebato de la personería gremial de la AGTSyP, la Sala II tampoco consideró que cabría un apercibimiento "real" en caso de negligencia de la UTA a presentarse en el expediente y en la audiencia de cotejo para hacer valer su representación, con lo que el sistema quedaría indefectiblemente cerrado a nuevos intentos de desplazamiento de personería gremial.

En un comunicado, y similarmente a lo expresado por Gianibelli (2017), los Metrodelegados calificaron el fallo de "escandaloso" y aseveraron que toda la sociedad reconoce a la AGTSyP como representante de los trabajadores del subte. No bastaría que el sindicato haya demostrado en cada oportunidad que es el sindicato con mayor cantidad de afiliados en el subte, "pero si premió a la UTA garantizándole que no concurrir a las audiencias le permite mantener su status [sic] en forma indefinida" (AGTSyP, 2017a). 
Calificó el fallo de "político", contribuyendo a la ofensiva del gobierno del presidente Mauricio Macri contra los trabajadores, utilizando el Poder Judicial para domesticarlos y así debilitar a las organizaciones sindicales, transfiriendo los recursos económicos de los sectores populares hacia los sectores con mayor poderío económico (Sindicato del Subte, 2017a).

A raíz de ello, los Metrodelegados interpusieron recurso extraordinario ante la Corte Suprema y alegaron, entre otras, la violación de normas de carácter federal como artículo 14 bis de la Constitución nacional (2010) y del Convenio 87 de la OIT (1948). Al mismo tiempo, en octubre de 2017, el gremio también había interpuesto una denuncia ante la Comisión Interamericana de Derechos Humanos. En este sentido, advirtió la interpretación restrictiva que realizó la Cámara de Apelaciones del Trabajo respecto de la adjudicación de la personería gremial y cerró toda posibilidad de controversia por mayor representatividad. Asevera la persecución y pedidos de desafuero sufridos por los representantes gremiales, tanto por parte de las empresas como del Estado (AGTSyP, 2017b).

Previo a la sentencia de la Corte Suprema, Enrique Rosito, secretario de prensa de la AGTSyP había dicho que

si bien es cierto que con este fallo de la Cámara el poder de firma del convenio colectivo de trabajo lo tiene la UTA, el gobierno se ha comprado un problema porque no puede garantizar el ejercicio real de este acuerdo paritario si no está previamente discutido con los que tienen el poder real, con la unanimidad que da la confianza de los compañeros, que somos nosotros. (AGTSyP, 2017c, el énfasis es nuestro)

La cita es interesante puesto que deja entrever que el conflicto se ejerce "de hecho", mediante el poder "real" de los trabajadores. La representación se ejerce independientemente de contar o no con la personería gremial. Sin perjuicio de ello, en aquel momento el sindicato estimaba que la Corte Suprema invalidaría tal decisión debido a lo escandaloso del fallo de la Cámara de Apelaciones del Trabajo (AGTSyP, 2017c).

Finalmente, en marzo de 2018 la Corte Suprema evitó expedirse sobre la cuestión planteada por los Metrodelegados. En este contexto, aseveró que la Sentencia 110121 no constituía una resolución definitiva y convocó a una nueva audiencia de cotejo en el Ministerio de Trabajo a fin de respetar las garantías procesales de la UTA, por lo que en la actualidad la AGTSyP no cuenta con la personería adquirida en 2015. Tal audiencia aún no se ha llevado a cabo (Sentencia CNT 77335/2014/CSI, 2018).

Sin perjuicio de ello, los Metrodelegados han remarcado que continúan siendo el sindicato más representativo y "contando con todos los derechos sindicales que ha venido ejerciendo desde antes del inicio del trámite de personería gremial, durante su transcurso, y en la actualidad" (AGTSyP, 2018a). Consideran la personería gremial 
como un "reconocimiento formal", y que no contar con él no impedirá la defensa de derechos de los trabajadores.

Siguiendo a Patricia Ventrici (2018), "el caso de los Metrodelegados es testimonio del boicot de la Corte Suprema a la democratización pendiente del mundo sindical. Al mismo tiempo, el Ministerio de Trabajo abusa de la intervención a sindicatos". Afirma el carácter estructural de este enfrentamiento que proviene tanto del Gobierno con los objetivos de flexibilización de la relaciones de trabajo como de las cúpulas de los grandes sindicatos tradicionales (como la UTA).

El fallo de la Corte Suprema implica un paso atrás para las expresiones alternativas al modelo de sindicalismo "burocrático", y aquello se evidencia aún más en el caso de los Metrodelegados, cuya actividad a lo largo de veinte años los posiciona como un interlocutor necesario en el servicio subterráneo. Sin perjuicio de ello y más allá de que la capacidad de conflicto se desarrolle en los hechos, la persecución institucional (desde diferentes actores estatales) es un escollo de gran importancia para el ejercicio pleno de los derechos sindicales, y es allí cuando el conflicto se convierte en el principal aliado, especialmente en el caso de sindicatos sin reconocimiento pleno por parte de las autoridades estatales.

\section{3. ¿CUÁL ES EL ROL DEL CONFLICTO?}

Casi al mismo tiempo de la publicación de la Sentencia CNT 77335/2014/CSl (2018) de la Corte Suprema, la UTA firmó un nuevo acuerdo paritario con Metrovías y el Gobierno de la Ciudad Autónoma de Buenos Aires². En este se estableció un aumento salarial del $15 \%$ en dos cuotas, en concordancia con los lineamientos y bases de negociación estatales de aquel año. A finales de mayo de 2018, en medio de un conflicto colectivo cuyo origen consistió en el reclamo de paritarias entre la AGTSyP y el gobierno de la Ciudad Autónoma de Buenos Aires. A ello se suma el pedido de revisión de formaciones con materiales cancerígenos, el asbesto.

Durante uno de los paros de actividades anticipados por los Metrodelegados, la policía bajó a los andenes de la línea $\mathrm{H}$, reprimió la medida de fuerza y detuvo a dieciséis trabajadores y delegados gremiales, entre los que figuraba Néstor Segovia, secretario general adjunto del gremio (Agencia de Noticias Red Acción, 2018). Es interesante que el gobierno de la ciudad de Buenos Aires considerara "ilegal" al gremio debido a que no contaba con la personería gremial, cuando aquella característica no impediría llevar a cabo medidas de fuerzas gremiales por cualquier gremio simplemente inscripto, de acuerdo con el artículo 14 bis de la Constitución nacional (2010) y el Convenio 87 de la OIT (1948). Esto quiere decir que, de acuerdo con aquellas normas, no es necesaria la personería gremial para ejercer

\footnotetext{
2 A partir del año 2011, el subterráneo pasa a ser de dirección compartida por Metrovías y el gobierno de la ciudad de Buenos Aires (a través de la empresa SBASE).
} 
medidas de acción directa, como una huelga. Sumado a ello, este no es derecho incluido en el artículo 31 de la Ley 23.551 (1988).

Sumado a ello, el Gobierno consideró que aquella medida de fuerza había constituido un atentado y resistencia a la autoridad policial, junto con la violación del Código Penal de la Nación (1921), más precisamente del artículo 194 que establece que quien impidiere, estorbare o entorpeciere el normal funcionamiento de los transportes por tierra, agua o aire o los servicios públicos de comunicación, de provisión de agua, de electricidad o de sustancias energéticas, "será reprimido con prisión de tres meses a dos años".

Por aquello años, la Corte Suprema de Justicia de la nación dicta la Sentencia 339:760 (2016) en la que se garantiza el ejercicio del derecho de huelga solo a los sindicatos reconocidos oficialmente por el Ministerio de Trabajo. Si bien este fallo no asevera que solo los sindicatos con personería gremial pueden ejercer la huelga, en ese contexto adverso los Metrodelegados lo resignifican al aseverar que ellos poseen un derecho de reclamo gremial por constituir un sindicato simplemente inscripto (AGTSyP, 2018c). En consecuencia, la AGTSyP utiliza aquella sentencia para legitimar su acción gremial que la es raíz de numerosas denuncias y detenciones de representantes del gremio.

A principios de junio de 2018, luego de la represión y detención de trabajadores de finales de mayo, el Ministerio de Trabajo, en conjunto con el Banco Central, estableció que ninguna entidad bancaria podrá debitar cuotas de afiliación o cualquier otro aporte a las organizaciones sindicales que no posean personería gremial. Para ello, se basó en el artículo 38 de la Ley 23.551 (1988). Dicha orden fue objeto de una denuncia ante la OIT por parte de la AGTSyP debido a que la medida atenta contra la libertad sindical al desfinanciar a un gremio que cuenta con la afiliación de un considerable y representativo grupo de trabajadores y contra la libertad individual del trabajador para disponer los débitos en su cuenta sueldo como mejor considere.

Por otro lado, pocos días después, el Juzgado de Primera Instancia 2 en lo contencioso-administrativo y tributario de la Ciudad Autónoma de Buenos Aires a cargo del Juez Gallardo, ordenó al Gobierno de Rodríguez Larreta negociar paritarias con los Metrodelegados. En esta obligación que cumplimentó meses después, se pactó un aumento salarial que superaba en el doble al del convenio de la UTA de marzo de 2018. Aquí es donde el rol del conflicto es más evidente porque, más allá de no contar con la personería gremial y a pesar de que la ley no lo estipula, el Estado y la empresa finalmente se dispusieron a negociar con los Metrodelegados.

\section{EL CONFLICTO POR EL ASBESTO EN EL SUBTERRÁNEO (2018-2019)}

En medio de los conflictos por paritarias el subte, atravesó otra cuestión de gran importancia con respecto de la salud de los trabajadores. Concretamente, las 
formaciones marca "CAF 5000" (adquiridos del Gobierno de España) correspondientes a la línea B, presentaban altos niveles de asbesto, sustancia volátil que puede generar severos problemas de salud e incluso cáncer.

Los Metrodelegados reclamaban investigaciones sobre esta sustancia desde marzo de 2018, al mismo tiempo que se publicaba la sentencia de la Corte Suprema respecto de la personería gremial. En aquella oportunidad, ya habían adoptado medidas de fuerza a raíz de la venta y adquisición directa por parte del gobierno de la ciudad de Buenos Aires y Metrovías (transacción que consideraron como "absolutamente escandalosa"3) de treinta y seis formaciones de trenes que habían sido eliminados de circulación por parte del Metro de Madrid.

También en 2018, los Metrodelegados habían denunciado la contaminación de los coches Fiat (de la línea A, B y principalmente la E), los General Electric (de la "E") y los Nagoya (que circulan por la línea C). Comenzó una campaña de negligencia sistemática por parte del Gobierno, en la que se aseveró que no había peligro e ignoró directamente los pedidos de información y análisis médicos por parte del sindicato. Finalmente, en diciembre de 2018, la empresa estatal SBASE reconoció la presencia de piezas con amianto en las formaciones.

Mucho antes de la admisión por parte de la empresa, la AGTSyP solicitó el análisis de las piezas de los CAF 5000 a la Universidad Nacional del Sur (UNS) de Bahía Blanca (Buenos Aires). Esta no solo confirma la presencia de asbesto en los trenes de la línea $\mathrm{B}$, sino que el material sigue apareciendo en piezas de otras flotas, como los coches Mitsubishi que se encuentran en circulación desde hace más de sesenta años. Sumado a todo ello, la UNS determinó que también hay contaminación en las formaciones Nagoya 300 de la línea C y también en la línea E, por lo que la situación era aún más complicada (AGTSyP, 2018d, 2019a).

A principios de junio de 2019, finalmente se retiraron de circulación numerosos trenes de la marca CAF 5000, que circulaban principalmente en la línea B, debido a una peligrosa presencia de amianto en los vagones y piezas. Posteriormente, el presidente de SBASE admitió, en una nota para la CNN, que no se habían leído los planos y la composición de los trenes contaminados, lo que confirma aún más la responsabilidad civil (e incluso penal) de los adquirentes de las formaciones (AGTSyP, 2019b).

En lo que respecta al conflicto en el lugar de trabajo, creemos necesario presentar una pequeña crónica sobre el estado del reclamo en el taller Rancagua de la línea B, a metros de la estación Federico Lacroze de la Ciudad Autónoma de Buenos Aires. Dicho taller constituye el colectivo de trabajadores más activo, a la vez que el primer precedente de conflicto y reclamo colectivo respecto de la presencia de asbesto en el subterráneo

3 Así se referían en la conferencia de prensa celebrada el 15 de marzo de 2018, a la que aludiremos en las próximas páginas. 
En el taller Rancagua trabajan diariamente alrededor de ciento sesenta personas, entre técnicos, electricistas, mecánicos, pintores, torneros y limpieza. El conflicto tuvo su origen durante los meses de octubre y noviembre de 2018 por la sospecha de una enfermedad pulmonar laboral de un trabajador del taller. Ello dio el puntapié inicial para una investigación más profunda sobre el estado de las formaciones del taller.

Ante la falta de respuesta y la confirmación de la presencia de amianto en las formaciones de la línea B, C y E, los trabajadores consideraron que su salud peligraba por el contacto directo con las piezas contaminadas, lo que acrecentaba la posibilidad de enfermedades ocasionadas por el asbesto. De este modo, desde febrero de 2019 los trabajadores del taller (en su mayoría afiliados a la AGTSyP), efectuaron retenciones de tareas y se negaron a manipular las piezas contaminadas con asbesto (En el Subte, 2019).

Por último, hacia finales de diciembre de 2019, la justicia administrativa de la Ciudad Autónoma de Buenos Aires emitió una medida cautelar a raíz de un reclamo ambiental planteado por la AGTSyP (2020) con la ayuda de los trabajadores de las líneas del subte y diversos profesionales y especialistas ${ }^{4}$ durante el mes de diciembre.

Allí ordenó inspecciones y toma de muestras a todas las flotas de la red de las que se sospechaba que contenían piezas con asbesto en el plazo de diez días hábiles y la prohibición de intervenir formaciones e instalaciones fijas pasibles de contener el material. También se determinó que los trabajadores afectados por la suspensión de tareas fuesen reubicados en otras tareas. A su vez, ordenó a Subterráneos de Buenos Aires (SBASE) y Metrovías que informen el listado de trabajadores que han desempeñado tareas en el ámbito del subterráneo en el período de cuarenta años previos a la promoción de la demanda con su correspondiente hoja de ruta laboral, incluyendo los actuales y los que por cualquier causa hayan cesado.

La resolución judicial indica la responsabilidad del gobierno de la ciudad, en cabeza de SBASE, por el conocimiento de que los trenes CAF 5000 contenían asbesto, ya que tal información constaba en los manuales de los trenes entregados por el Metro de Madrid, lo que evidenció la escasa o nula sensibilidad en materia de ambiente (AGTSyP, 2020).

¿Ahora bien, qué tiene que ver la personería gremial con todo esto? Incluso antes de adquirirla a finales de 2015, los Metrodelegados consideraban que dichos derechos exclusivos posibilitarían a los trabajadores que sus legítimos representantes ejerciesen plenamente los derechos colectivos a través de la firma de nuevos convenios colectivos. Por otro lado, estimaban que era "el último paso en la unificación de las representaciones real y formal en el ámbito del subterráneo" (AGTSyP, 2013a).

Con esto queremos decir que la representatividad del sindicato se observa desde dos lentes: uno "real" y otro "legal". El real lo ejercieron siempre, desde su misma

${ }_{4}$ Entre ellos, ingenieros, médicos, psicólogos y abogados. 
constitución en 2008, como una necesidad de controvertir el orden impuesto por la dirigencia de la UTA. Por otro lado, podríamos decir que la personería constituye una cuestión "accesoria" (pero necesaria) a los fines de acordar mayores derechos laborales (AGTSyP, 2013b).

Esto se evidencia aún más en la conferencia de prensa que ofrecieron los dirigentes sindicales a raíz de la sentencia de la Corte Suprema el día 15 de marzo de 20185. En aquella oportunidad Roberto Pianelli, secretario general del sindicato, consideró que el fallo es de "índole político", y cuyo fin es deslegitimar la lucha llevada a cabo por la AGTSyP en pos del mejoramiento del trabajo (AGTSyP, 2018b). En este sentido, favoreció a los sindicatos limitativos de los derechos de los trabajadores del subterráneo y firmó convenios "a la baja" (esto es, limitados y con bajos beneficios).

Ricardo Pianelli agrega con convicción que "los únicos representantes de los trabajadores del subte es este sindicato" (AGTSyP, 2018b). Refuerza su opinión diciendo que van a continuar ejerciendo la representatividad de los trabajadores del subterráneo, como siempre lo hicieron a lo largo de toda su historia. Este fallo es un intento de amedrentamiento de los trabajadores que anticipó que no va a dar resultado. No hay trámite burocrático que impida ejercer la representación, como lo vienen haciendo desde "hace casi 20 años" (AGTSyP, 2018b).

Concretamente, lo más importante no es el fallo de la Corte. Para ellos no cambia nada, es solo un papel, un trámite. La verdadera fuerza es su legitimidad en los hechos, en la representatividad. Por ello, la conferencia de prensa termina siendo en reclamo de la salud de los trabajadores que podrían estar enfermos por el asbesto y no por la sentencia en sí misma. El fallo no los amedrentaría, no impediría sus medidas de acción directa. Un "papel" no cambia las acciones de los trabajadores a lo largo de los años. Por otro lado, Néstor Segovia, secretario general adjunto, asevera que el Gobierno pensaba que ellos iban a parar por el fallo, pero les aclara vehementemente que ellos paran por reclamos de los trabajadores y que no necesitan "ninguna chapa" para representarlo, refiriéndose al trámite de personería gremial (AGTSyP, 2018b).

Con estas notas de campo que hemos tomado sobre la conferencia de prensa, señalamos que las exposiciones, tanto de los dirigentes como de los demás políticos y personalidades que los apoyaban, no se centraron en la sentencia y sus implicancias prácticas, sino que el problema del asbesto fue otra de las maneras de continuar afianzando una representación "real" de los trabajadores del subte en un tema tan delicado como la salud laboral y pública. Esto quiere decir que el fallo fue una "excusa" para decir "aquí estamos y seguiremos estando" más allá del "papel", de la "chapa" y

Realizamos notas de campo de tipo etnográfico sobre la conferencia de prensa, las citas textuales están entre comillas. La conferencia de prensa se encuentra disponible en la página de Facebook del órgano de prensa de la AGTSyP (2018b). 
de las sentencias judiciales que benefician a sindicatos que, de acuerdo con las notas, no representan los intereses colectivos de los trabajadores.

El conflicto del asbesto es de gran importancia puesto que afianza dos ideas principales: la reafirmación del rol de la AGTSyP como interlocutor necesario y como articulador de los reclamos laborales, en este caso de la salud. Segundo, el rol del conflicto en el taller Rancagua (en su gran mayoría afiliados a la AGTSyP) que se convierte una vez más en la piedra angular para el mejoramiento de las condiciones de trabajo más allá de la personería gremial como instituto jurídico.

\section{CONCLUSIONES}

Mediante estas líneas, intentamos ofrecer un panorama lo más actualizado del estado de la personería gremial como medio para la adquisición y afianzamiento de derechos a partir del caso de los Metrodelegados del subterráneo. Junto con ello, y relacionado con su derrotero histórico y constitución como colectivo gremial independiente, analizamos el modo en que el conflicto actúa frente a esta cuestión y la legitimidad que ofrece en un contexto de pérdida "formal" de representación colectiva.

Vimos cómo la personería gremial excede lo meramente administrativo o jurídico para convertirlo en una cuestión de prioridades del Estado, quien a través del Ministerio de Trabajo o de la Justicia Nacional del Trabajo se constituye en este caso en una traba al pleno ejercicio de los derechos colectivos. Pero ante los embates políticos y aparentemente "institucionales", como la falta de cumplimiento del procedimiento de compulsa de afiliados, derecho de defensa, ilegalidad de la huelga o paro por parte del Estado, la respuesta también es política. Y es allí donde el conflicto pasa a un primer plano y se independiza de la norma, siempre actuando dentro de reclamos de gran trascendencia e importancia, como en el caso del asbesto.

El caso del asbesto es paradigmático porque es uno de los últimos y más vastos ejemplos de las implicancias y contradicciones entre lo normativo y el conflicto. Aquí también estamos en presencia de un sindicato sin reaseguros formales que garanticen la representación. Sin embargo, el conflicto genera un estado de cambio permanente que fuerza la toma de medidas, incluso en temas tan sensibles como la salud y las condiciones de trabajo.

Es decir, ¿qué es más importante que la representación de los trabajadores en materia no solo laboral, sino pública? Por ello, pensamos que el tema de personería gremial y el conflicto colectivo es solo una de las aristas que puede asumir el binomio de representación-representatividad en las relaciones colectivas de trabajo. Aquí se manifiesta tanto en la retención de tareas por parte de los trabajadores de taller Rancagua como en la intensa campaña del sindicato para visibilizar esta cuestión que, como dijimos, no solo incumbe a los trabajadores y trabajadoras del subte, sino que afecta a la sociedad en su conjunto. 


\section{REFERENCIAS}

Afarian, J. y Pasarín, S. (2019). La ley sobre convenciones colectivas de trabajo de 1953 y la opinión sindical en su tratamiento parlamentario. Discusiones tempranas del "modelo sindical argentino. Anuario del Instituto de Historia Argentina, 19(2), 1-16. https://doi.org/10.24215/2314257Xe101.

Afarian, J. (2019b). Y, para colmo, el asbesto. Movimiento, 13. http://www.revistamovimiento.com/ politicas/y-para-colmo-el-asbesto/

Afarian, J. (2019a). La espera de la personería gremial como artefacto disciplinador de la lucha gremial en el subterráneo. Movimiento, 8. http://www.revistamovimiento.com/politicas/la-espera-de-lapersoneria-gremial-como-artefacto-disciplinador-de-la-lucha-gremial-en-el-subterraneo/

Afarian, J. (2018, 20 de marzo). Luchas Subterráneas: los "Metrodelegados" frente a la precarización laboral [ponencia]. Seminario Internacional sobre Tercerización Laboral, Buenos Aires, Argentina.

Agencia de Noticias Red Acción (2018, 22 de mayo). Represión contra los trabajadores del subte. ANRed. https://www.anred.org/2018/05/22/represion-contra-los-trabajadores-del-subte/

Arias, C.C. y Haidar, V. (2008). Resistir en nombre de la salud. Un análisis de la experiencia del Cuerpo de Delegados de los subterráneos de Buenos Aires. Trabajo y Sociedad, 11, 1-11. https://www.unse. edu.ar/trabajoysociedad/ARIAS_HAIDAR.pdf

Arias, C.C., Diana Menéndez, N. y Salgado, P.D. (2013). Los desafíos de construir institucionalidad: el sindicato del subte de Buenos Aires. Espacio Abierto: Cuaderno Venezolano de Sociología, 22(3), $455-$ 475. https://www.redalyc.org/articulo.oa?id=12228905005

Arias, C. C. (2015), Tercerización en el subterráneo de Buenos Aires: prácticas empresarias y disputas sindicales [ponencia]. XII Congreso Nacional de Estudios del Trabajo, Buenos Aires, Argentina. https://www. aset.org.ar/2015/ponencias/9_Arias.pdf

Asociación Gremial de Trabajadores del Subte y Premetro, AGTSyP (2020, 29 de enero). La Justicia ordena inspeccionar todas las flotas del Subte en 10 días por el asbesto. Sindicato del Subte. http://sindicatodelsubte. com.ar/?La-Justicia-ordena-inspeccionar-todas-las-flotas-del-Subte-en-10-dias-por-el

Asociación Gremial de Trabajadores del Subte y Premetro, AGTSyP (2019b, 15 de octubre). El titular del Subte reconoció que la presencia de asbesto estaba en los manuales. Sindicatos del Subte. http://sindicatodelsubte. com.ar/?El-titular-del-Subte-reconocio-que-la-presencia-de-asbesto-estaba-en-los

Asociación Gremial de Trabajadores del Subte y Premetro, AGTSyP (2019a, 6 de mayo). La Línea B en estado naranja por el asbesto y la flota. Sindicato del Subte. http://sindicatodelsubte.com.ar/?LaLinea-B-en-estado-naranja-por-el-asbesto-y-la-flota

Asociación Gremial de Trabajadores del Subte y Premetro, AGTSyP (2018d, 4 de julio). Informes Universidad de Sur, Asbesto Positivo en Flotas Mitsubishi, GEE, Nagoya. Sindicato del Subte. http://sindicatodelsubte. com.ar/?Informes-Universidad-de-Sur-Asbesto-Positivo-en-Flotas-Mitsubishi-GEE-Nagoya

Asociación Gremial de Trabajadores del Subte y Premetro, AGTSyP (2018c, 10 de abril). Ante supuestas denuncias del Ministerio de Trabajo a este sindicato. Sindicato del Subte. http://sindicatodelsubte.com. ar/?ANTE-SUPUESTAS-DENUNCIAS-DEL-MINISTERIO-DE-TRABAJO-A-ESTE-SINDICATO

Asociación Gremial de Trabajadores del Subte y Premetro, AGTSyP (2018b, 15 de marzo). Conferencia de prensa ante el escandaloso fallo de la Corte Suprema. Prensa del Subte. https://www.facebook.com/ prensadelsubte/videos/834814483387385/ 
Asociación Gremial de Trabajadores del Subte y Premetro, AGTSyP (2018a, 14 de marzo). La AGTSyP $y$ sus derechos sindicales. Sindicato del Subte. http://sindicatodelsubte.com.ar/?LA-AGTSyP-Y-SUSDERECHOS-SINDICALES

Asociación Gremial de Trabajadores del Subte y Premetro, AGTSyP (2017c, 14 de marzo). Fallo contra los Metrodelegados: "En la Corte Suprema va a ser invalidado". Sindicato del Subte. http://sindicatodelsubte. com.ar/?Fallo-contra-los-Metrodelegados-En-la-Corte-Suprema-va-a-ser-invalidado

Asociación Gremial de Trabajadores del Subte y Premetro, AGTSyP (2017b, 24 de octubre). Denuncia ante la CIDH por atropellos a derechos laborales. Sindicato del Subte. http://sindicatodelsubte.com. ar/?Denuncia-ante-la-CIDH-por-atropellos-a-derechos-laborales

Asociación Gremial de Trabajadores del Subte y Premetro, AGTSyP (2017a, 10 de marzo). Insólito fallo contra los Metrodelegados. Sindicato del Subte. http://sindicatodelsubte.com.ar/?INSOLITO-FALLOCONTRA-LOS-METRODELEGADOS

Asociación Gremial de Trabajadores del Subte y Premetro, AGTSyP (2016, 8 de junio). Rechazo al fallo de la Corte Suprema sobre derecho de huelga. Sindicato del Subte. http://sindicatodelsubte.com. ar/?RECHAZO-AL-FALLO-DE-LA-CORTE-SUPREMA-SOBRE-DERECHO-DE-HUELGA

Asociación Gremial de Trabajadores del Subte y Premetro, AGTSyP (2013b, 27 de noviembre). Un paso más hacia la obtención de la personería gremial. Sindicato del Subte. http://sindicatodelsubte.com. ar/?UN-PASO-MAS-HACIA-LA-OBTENCION-DE-PERSONERIA-GREMIAL

Asociación Gremial de Trabajadores del Subte y Premetro, AGTSyP (2013a, 1 de agosto). Se presentó la solicitud de personería gremial para la AGTSyP. Sindicato del Subte. http://sindicatodelsubte.com. ar/?SE-PRESENTO-LA-SOLICITUD-DE-PERSONERIA-GREMIAL-PARA-LA-AGTSYP

Asociación Gremial de Trabajadores del Subte y Premetro, AGTSyP (2009b, 12 de noviembre). Reunión con el Ministerio de Trabajo por la inscripción gremial de AGTSyP. Sindicato del Subte. http:// sindicatodelsubte.com.ar/?REUNION-EN-EL-MINISTERIO-DE-TRABAJO-POR-LA-INSCRIPCIONGREMIAL-DE-AGTSYP

Asociación Gremial de Trabajadores del Subte y Premetro, AGTSyP (2009a, 19 de noviembre). Por nuestra personería, apostamos al diálogo. Sindicato del Subte. http://sindicatodelsubte.com.ar/?PORNUESTRA-PERSONERIA-APOSTAMOS-AL-DIALOGO

Basualdo, V., Esponda, A., Gianibelli, G. y Morales, D., (2015). Tercerización y derechos laborales en la Argentina actual. Flacso-CELS, Universidad Nacional de Quilmes.

Biblioteca del Congreso de la Nación (2010). Constitución de la Nación Argentina. Buenos Aires; BCNCSJN. https://bibliotecadigital.csjn.gov.ar/Constitucion-de-la-Nacion-Argentina-Publicacion-delBicent.pdf

Bouvet, V. (2008). Un fantasma recorre el subte: crónica de la lucha de los trabajadores de Metrovías. Desde el Subte Editorial.

Cámara Nacional de Apelaciones de Trabajo, Sala II (2017, 6 de marzo). Sentencia 110121 (Ministerio de Trabajo y Seguridad Social c. Asociación Gremial de Trabajadores del Subterráneo y Premetro y otros s/ ley de asociaciones sindicales). http://www.saij.gob.ar/camara-nacional-apelacionestrabajo-nacional-ciudad-autonoma-buenos-aires-ministerio-trabajo-seguridad-social-asociaciongremial-trabajadores-subterraneo-premetro-otros-ley-asoc-sindicales-fa 17040002-2017-0306/123456789-200-0407-1ots-eupmocsollaf 
Castillo, C., Collado, A., Elbert, R., Feijoó, C., Ricciardelli, C., Rouspil, C. y Stricker, D. (2007), Experiencias subterráneas. Trabajo, organización gremial e ideas políticas de los trabajadores del subte. Ediciones del IPS.

Congreso de la Nación Argentina (1921, 29 de octubre). Código Penal de la Nación Argentina. Actualizado 2021. Infoleg. Información Legislativa. http://servicios.infoleg.gob.ar/infolegInternet/ anexos/15000-19999/16546/texact.htm

Congreso de la Nación Argentina (1953, 29 de septiembre). Ley 14.250 de 1953. Convenios Colectivos de Trabajo. Boletín Oficial de la Nación Argentina n. ${ }^{\circ}$ 17507. http://servicios.infoleg.gob.ar/ infolegInternet/verNorma.do?id=46379

Congreso de la Nación Argentina (1988, 23 de marzo). Ley 23.551 de 1988. Régimen de Asociaciones Sindicales. Boletín Oficial de la Nación Argentina n 26366. http://servicios.infoleg.gob.ar/infolegInternet/ verNorma.do?id $=20993$

Congreso de la Nación Argentina (1989, 17 de agosto). Ley 23.696 de 1989. Reforma del Estado. Emergencia Administrativa. Boletín Oficial de la Nación Argentina n²6702. http://servicios.infoleg.gob.ar/ infolegInternet/verNorma.do?id $=98$

Congreso de la Nación Argentina (1991, 13 de noviembre). Ley 24.013 de 1991. Ley de Empleo. Protección del Trabajo. Boletín Oficial de la Nación Argentina n² 27286. http://servicios.infoleg.gob.ar/ infolegInternet/verNorma.do?id=412

Corte Suprema de Justicia de la Nación (2018, 13 de marzo). Sentencia CNT 77335/2014/CSl/2018 (Ministerio de Trabajo y Seguridad Social c. Asociación Gremial de Trabajadores del Subterráneo y Premetro y otros s/ ley de asociaciones sindicales). https:/www.cij.gov.ar/nota-29507-LaCorte-Suprema-deja-firme-el-fallo-que-declar--la-nulidad-de-la-personer-a-gremial-otorgada-alsindicato-de-los-trabajadores-del-subterr-neo.html

Corte Suprema de Justicia de la Nación (2016, 7 de junio). Sentencia 339:760/2016 (Orellano, Francisco Daniel c. Correo Oficial de la República Argentina S.A. s/ juicio sumarísimo). https://www.cij.gov. ar/nota-2 1852-La-Corte-resolvi- que-solo-los-gremios-tienen-el-derecho-de-promover-huelgasy-que-los-grupos-informales-de-trabajadores-no-pueden-promover-medidas-de-fuerza.html

Corte Suprema de Justicia de la Nación (2009, 09 de diciembre). Sentencia 332.2715/09 (Rossi c. Estado Nacional-Armada Argentina). http://www.saij.gob.ar/corte-suprema-justicia-nacion-federal-ciudadautonoma-buenos-aires-rossi-adriana-maria-estado-nacional-armada-argentina-sumarisimofa09000 115-2009-12-09/123456789-511-0009-0ots-eupmocsollaf

Corte Suprema de Justicia de la Nación (2008, 11 de noviembre). Sentencia 331:2499/08 (Asociación Trabajadores del Estado c. Ministerio de Trabajo s/ ley de asociaciones sindicales). http://www. saij.gob.ar/corte-suprema-justicia-nacion-federal-ciudad-autonoma-buenos-aires-asociaciontrabajadores-estado-ministerio-trabajo-ley-asociaciones-sindicales-fa08000261 -2008- 11 11/123456789-162-0008-0ots-eupmocsollaf

Corte, N. (1994). El modelo sindical argentino. Régimen legal de las asociaciones sindicales. Santa Fe; RubinzalCulzoni Editores.

Cortés, R. y Marshall, A. (1991). Estrategias económicas, intervención social del Estado y regulación de la fuerza de trabajo. Estudios del Trabajo, 1, 21-46. https://bit.ly/3kbD4fO

Cresto, J. (2012). El proceso de autonomización sindical de los trabajadores del Subterráneo de Buenos Aires. Conformación y desarrollo de la Asociación Gremial de Trabajadores del Subterráneo y 
Premetro (AGTSyP). Conflicto Social, 5(7), 12-42. https://publicaciones.sociales.uba.ar/index.php/ CS/article/view/316

Cresto, J. (2010). La organización gremial de los trabajadores del subterráneo de Buenos Aires y su resistencia a la ofensiva de la concesionaria sobre la fuerza de trabajo (1994-2001). Documentos de Jóvenes Investigadores, 23, 1-55. http://biblioteca.clacso.edu.ar/Argentina/iigg-uba/201 1031 1043739/ ji23.pdf

Dantón Bermúdez, M.A. (2013, 27, 28 y 29 de noviembre). 'Estamos pariendo un sindicato': debates en torno al proceso de organización sindical en el subte de Buenos Aires, [ponencia]. VII Jornadas Santiago Wallace de Investigación en Antropología Social, Buenos Aires, Argentina. http://www.aacademica. org/000-063/461

Drolas, A. (2003, 13-16 de agosto). Los enigmas de la representación: una mirada sobre el sindicato [ponencia]. VI Congreso Nacional de Estudios del Trabajo, Buenos Aires, Argentina. https://www.aset.org.ar/ congresos/6/archivosPDF/grupoTematico03/004.pdf

En el Subte (2019, 8 de mayo). Crisis en la línea B: trabajadores se niegan a reparar coches con asbesto. En el Subte.com. https://enelsubte.com/noticias/crisis-en-la-linea-b-trabajadores-se-niegan-a-repararcoches-con-asbesto/

Gianibelli, G. (2017, 11 de marzo). Subte: Otra vez, el muro. Argentina Indymedia. https://archivo.argentina. indymedia.org/news/2017/03/903620.php

Ministerio de Trabajo, Empleo y Seguridad Social de la Nación (2015, 1 de diciembre). Resolución 1601/15. Otórgase Personería Gremial. Boletín Oficial de la Nación. https://www.boletinoficial.gob. ar/detalleAviso/primera/137200/20151201

Montes Cató, J.S. y Ventrici, P. (2010). El lugar de trabajo como espacio de resistencia a las políticas neoliberales. Reflexiones a partir de las experiencias de los trabajadores telefónicos y del subte. Revista Theomai, 22, 101-119. http://revista-theomai.unq.edu.ar/numero\%2022/Art_VentriciCat\%C3\%B3.pdf

Organización Internacional del Trabajo (2006). La Libertad Sindical. Recopilación de decisiones y principios del Comité de Libertad Sindical del Consejo de Administración de la OIT. OIT.

Organización Internacional del Trabajo (1949, 8 de junio). Convenio 98 de 1949. Convenio sobre el derecho de sindicación y de negociación colectiva. https://www.ilo.org/dyn/normlex/es/f?p=NORMLEXPUB: 1210 0:0::NO::P12100_ILO_CODE:C098

Organización Internacional del Trabajo (1948, 17 de junio). Convenio 87 de 1948. Convenio sobre la libertad sindical y la protección del derecho de sindicación. https://www.ilo.org/dyn/normlex/es/f?p= NORMLEXP UB: $12100: 0:: N O:: P 12100 \_I N S T R U M E N T \_I D: 312232$

Poder Ejecutivo de la Nación (1988, 14 de abril). Decreto 467/88. Apruébase la reglamentación de la Ley $\mathrm{N}^{\circ}$ 23.551. Infoleg. Información Legislativa. http://servicios.infoleg.gob.ar/infolegInternet/ anexos/45000-49999/46044/norma.htm

Poder Ejecutivo de la Nación (1945, 13 de octubre). Decreto 23.852. Apruébase el régimen el régimen legal de las asociaciones profesionales de trabajadores. Boletín Oficial de la Nación. https://www.boletinoficial. gob.ar/detalleAviso/primera/7027955/19451013

Pontoriero, E. (2015). Estado de excepción y contrainsurgencia: el plan Conintes y la militarización de la seguridad interna en Argentina. Contenciosa, 3(4), 1-16. http://www.contenciosa.org/Sitio/ VerArticulo.aspx?i $=40$ 
Recalde, M. (2015). El modelo sindical argentino. Régimen jurídico. Editorial Eduvim.

Salas, E. (1990). La huelga del Frigorífico Lisandro de la Torre. Editorial Seal.

Trajtemberg, D., Senén González, C., Borroni, C. y Pontoni, G. (2012, 5, 6 y 7 de diciembre). Representación sindical en los lugares de trabajo: un análisis del Módulo de Relaciones Laborales [ponencia]. VII Jornadas de Sociología de la UNLP, Buenos Aires, Argentina. http://nulan.mdp.edu.ar/2249/1/trajtemberg. etal.2012.pdf

Trajtemberg, D. y Battistini, O. (2015). Representación sindical en Argentina: Un caso fuera de modelo. Quaestio Juris, 8(1), 386-419. http://dx.doi.org/10.12957/rqi.2015.15365

Tribuzio, J.E. (2012, 10, 11 y 12 de mayo). ¿Un Nuevo Modelo Sindical Argentino? [ponencia]. XIX Congreso Nacional de Derecho del Trabajo y de la Seguridad Social, Buenos Aires, Argentina. https://www.aadtyss.org.ar/old/congresos/ponencias/Jose\%20E.\%20Tribuzio.pdf

Ventrici. P. (2018, 26 de marzo). Sindicalismo y cambio de época. Sindicato del Subte. http://sindicatodelsubte. com.ar/?Sindicalismo-y-cambio-de-epoca

Ventrici, P. (2016). La crisis del modelo sindical. Proyectos para la modificación de la Ley de Asociaciones Sindicales. Ciencia, Docencia y Tecnología, 52(27), 73-105. http://www.pcient.uner.edu. ar/cdyt/article/view/123

Ventrici, P. (2011). Sindicalismo de base en la Argentina contemporánea. El cuerpo de delegados del subterráneo [tesis de doctorado no publicada, Universidad de Buenos Aires]. Repositorio Institucional UBA.

Ventrici, P., Compáñez, M. y Vocos, F. (2012). Metrodelegados. Subte: de la privatización al traspaso. Desde el Subte Editorial.

Verbitsky, H. y Bohoslavsky, E. (2013). Cuentas pendientes. Los cómplices económicos de la dictadura. Siglo Veintiuno Editores.

Vocos, F. (2008). Enfrentando la Ofensiva Empresaria: La Construcción del Anteproyecto de Convenio Colectivo por los Trabajadores del Subte. Labour Again Publications, 1-30. http://www.iisg.nl/ labouragain/documents/vocos.pdf 